\title{
Molecular orbital investigation of chemisorption. I. Hydrogen on tungsten (100) surface
}

\author{
Leon W. Anders and Robert S. Hansen \\ Ames Laboratory-USAEC and Department of Chemistry, Iowa State University, Ames, Lowa 50010
}

L. S. Bartell

Department of Chemistry, University of Michigan, Ann Arbor, Michigan 48104

(Received 6 June 1973)

\begin{abstract}
The relative bonding energies of hydrogen chemisorbed at three symmetric sites on a $W(100)$ surface were obtained by means of the extended Hückel molecular orbital theory (EHMO). The preferred site for hydrogen chemisorption was found to be the single coordination number $(1 \mathrm{CN})$ site or the site above a surface tungsten atom. The $\mathrm{W}(100)$ surface was represented by finite arrays of tungsten atoms which were shown to be adequate for obtaining semiquantitative results. The basis set for the calculations contained the valence orbitals of tungsten and, initially, the $5 p$ orbitals which were nonbonding but provided the necessary repulsion at small internuclear separation. The repulsive energy provided by these orbitals was replaced by an analytical exponential repulsive energy term. This allowed the $5 p$ orbitals to be omitted from the basis set to simplify computation. Functionally, the energy change for the reaction $\mathrm{W}_{n}+\mathrm{H}-\mathrm{W}_{n} \mathrm{H}$ was calculated for various assumed configurations of the $\mathrm{W}_{n} \mathrm{H}$ "molecule." The bonding between tungsten atoms was found to be changed as a result of $\mathbf{W}_{n} \mathbf{H}$ formation, and the change varied with hydrogen position. Energy barriers to surface diffusion were also calculated and found to agree reasonably with experimental values.
\end{abstract}

\section{INTRODUCTION}

In recent years, it has become experimentally possible to study the chemisorption of atoms and molecules on single crystal surfaces. To account for the experimental observations from these welldefined surfaces, various bonding models have been proposed, but the detailed description of the adsorbed species remains in the realm of speculation. Unfortunately, no available experimental techniques give conclusive information about the molecular structures or site distributions of the adsorbed species. In view of current experimental limitations and the importance of the implications in the field of catalysis, a theoretical attack seems justified. The prohibitive expense of $a b$ initio quantum mechanical calculations for such systems make it attractive to explore nonrigorous, semiempirical methods which have been found in the past to account quite well for structural features of molecular systems.

In this paper, we apply the extended Hückel molecular orbital theory, a semiempirical molecular orbital technique, to investigate the preferred structure of the "surface molecule." EHMO has been used previously to study the chemisorption of hydrogen on graphite, ${ }^{1}$ and of organic molecules, ${ }^{2}$ carbon monoxide, ${ }^{3}$ and hydrogen ${ }^{4}$ on nickel. In the case of carbon monoxide on nickel, ${ }^{3}$ the dependence of binding energy on distance at the possible chemisorption sites was not analyzed. The preferred site was selected by comparison of binding energies at a fixed carbon-nickel distance. These investigations used only the valence orbitals in the basis set, and as a result only the hydrogen- graphite interaction produced a minimum in the binding energy curve. The interaction of hydrogen as well as carbon, nitrogen, oxygen, and fluorine on graphite ${ }^{5}$ was also investigated using CNDO technique in order to alleviate the problem of unreasonable charge transfer which can occur in the EHMO calculations.

Although it is known that this technique has many limitations, it nevertheless has been successful in determining preferred molecular configurations. Therefore, it is a promising tool for investigating the structure of the elusive surface molecules and for obtaining a better understanding of the bonding between chemisorbed atoms and the surface atoms. We have chosen to investigate the chemisorption of hydrogen on a tungsten (100) surface because of the experimental data which are available for comparison. ${ }^{6-11}$

\section{PROCEDURE}

Hoff mann ${ }^{12}$ developed a semiempirical molecular orbital theory, including all overlap integrals, referred to as the "extended Hückel" molecular orbital theory (EHMO). It was originally utilized to solve organic conformational problems, but subsequently has been used to yield insight on other molecular geometric problems, including the structural chemistry of crystals. ${ }^{13}$ Hoffmann stressed that the method's most reliable characteristic is its ability to predict molecular geometries and to assess the relative importance of sigma and pi orbitals. On the other hand, it is quite crude in predicting charge distributions and excitation energies in electronic transitions. 
The molecular orbitals are formed from a linear combination of atomic orbitals (LCAO),

$$
\Psi_{j}=\sum_{q} C_{j q} \chi_{a},
$$

where the atomic orbitals $\chi_{q}$ are the single parameter, Slater-type atomic orbitals (STO). According to the standard variation principle the LCAO function with $q$ atomic orbitals leads to a set of $q$ simultaneous linear homogeneous equations, each of which contains $q$ coefficients $C_{j q}$,

$$
\sum_{q}\left(H_{p q}-\epsilon_{j} S_{p q}\right) C_{j q}=0 \text {. }
$$

For these equations to have nontrivial solutions, the determinant of the coefficients must vanish,

$$
\left\|H_{p q}-\epsilon_{j} S_{p q}\right\|=0 \text {. }
$$

The eigenvalues $\epsilon_{j}$ given by the solution of this determinant are the energies of the molecular orbitals. These energies $\epsilon_{j}$ are substituted back into the set of simultaneous linear homogeneous equations which are then solved to give the coefficients $C_{j q}$. These coefficients $C_{j q}$ form the wavefunction of the molecular orbital at each energy $\epsilon_{j}$. In the Hoffmann formalism, the diagonal elements $H_{p p}$ are set equal to the valence orbital ionization potential (VOIP) of $\chi_{p}$; the nondiagonal elements $H_{p q}$ were approximated by the Mulliken-Wolfsberg-Helmholtz approximation $^{14}$

$$
H_{p q}=0.5 K\left(H_{p p}+H_{q q}\right) S_{p q},
$$

with a Helmholtz factor $K$ equal to 1.80 .

The EHMO method does not explicitly account for either electron-electron or core-core interactions between atoms, but relies on extensive cancellations which hold approximately for nonpolar molecules. These cancellations will also hold for other molecules near the equilibrium internuclear separations where the chemical bond is formed. Excessive charge transfers, a common problem in the EHMO method, were partially alleviated by adjusting the VOIP as functions of atomic charge $q$ and iterating to self-consistency. The atomic charge was computed by the Mulliken meth$\mathrm{od}^{15}$ and the VOIP of the atomic orbitals were de- termined as functions of charge $q$ according to the following ${ }^{16}$ :

$$
\operatorname{VOIP}(q)=A q^{2}+B q+C .
$$

These adjusted VOIP gave more realistic charge transfers than fixed VOIP. Bonding energies for different structures in the neighborhood of their energy minima were similarly shifted by shifts in VOIP so that conclusions as to preferred sites were unaffected by variations in VOIP.

Table I lists the orbital exponents for the STO and the values for the constants, $A, B$, and $C$, which are needed to determine the VOIP as a function of atomic charge. The values for the hydrogen $1 s$ orbital were obtained from Basch, Viste, and Gray. ${ }^{16}$ A value of 1.20 was used for the orbital exponent of the hydrogen $1 s$ orbital. For the tungsten $5 p$ orbital, the value of $C$ was obtained from the tables of Herman and Skillman ${ }^{17}$; the orbital exponent for the STO was obtained by the rules of Burns ${ }^{18}$ and assumed to be charge independent. The values of $C$ and the orbital exponents for the STO of the tungsten $5 d$ and $6 s$ orbital were obtained from Lohr and Lipscomb ${ }^{19}$; the values of $C$ for these orbitals agreed with neutral atom ionization measurements of Lotz. ${ }^{20}$ A linear charge dependence ${ }^{21}$ for the VOIP of the tungsten $5 d$ and $6 s$ orbitals was used with the value of $B$ equal to $3.5 \mathrm{eV} / e^{-}$.

The EHMO method was first applied to the diatomic molecules, $\mathrm{W}-\mathrm{W}$ and $\mathrm{W}-\mathrm{H}$. These calculations showed that it was necessary to include the inner orbitals of the tungsten atoms as well as the valence orbitals in the basis set to obtain a characteristic potential energy curve; when only valence orbitals were used too few nonbonding orbitals were populated to provide the requisite repulsion at small internuclear separations. It was found that the addition of the inner orbitals overcame this deficiency, although they made little contribution to the formation of the chemical bond. Therefore, if the repulsive energy could be calculated by an empirical relationship, these orbitals could be omitted, thus reducing the size

TABLE I. Atomic orbital parameters for extended Hückel molecular orbital calculations. Orbital exponents are for Slater type orbitals. Valence orbital ionization potential (VOIP) parameters reflect dependence of VOIP on charge $q$ according to VOIP $=A q^{2}+B q+C$.

\begin{tabular}{lccccc}
\hline \hline Atom & Orbital & Orbital exponent & $\begin{array}{c}A \\
\left(\mathrm{eV} /|e|^{2}\right)\end{array}$ & $\begin{array}{c}B \\
(\mathrm{eV} /|e|)\end{array}$ & $\begin{array}{c}C \\
(\mathrm{eV})\end{array}$ \\
\hline Hydrogen & $1 s$ & 1.20 & 13.60 & 27.20 & 13.60 \\
Tungsten & $5 p$ & 4.91 & 0.00 & 0.00 & 46.07 \\
& $5 d$ & 2.20 & 0.00 & 3.50 & 9.00 \\
& $6 s$ & 1.40 & 0.00 & 3.50 & 8.00 \\
\hline
\end{tabular}


of the basis set and saving a substantial computation effort. The repulsive energy between atoms in a molecule can be represented approximately by a series of exponential terms of the form,

$$
E_{\text {rep }}=\sum_{i j} A_{i j} e^{-r_{i j} / \alpha_{i j}},
$$

where the parameters, $A_{i j}$ and $\alpha_{i j}$, are unique for a pair of atoms. These parameters for pairwise interactions were derived from the repulsive energy, $E_{\text {rep }}$, of the diatomic molecules. The repulsive energy was determined as the difference in bonding energies obtained by a calculation with only valence orbitals and by a calculation which also included the inner orbitals. Comparison of $E_{\text {rep }}$ for $\mathrm{WH}$ and $\mathrm{W}_{2} \mathrm{H}$ indicated that repulsive energies in the tungsten-hydrogen system could be assumed pairwise additive.

In modeling the tungsten (100) surface for the EHMO calculations, it was necessary to truncate the infinite surface to a limited number of metal atoms. These selected surface arrays must represent the possible sites for bonding of a chemisorbed atom. The tungsten (100) appears to offer three distinct symmetric sites which might be considered attractive adsorption sites. These are shown in Fig. 1, which also shows the metal atom arrays used in calculations. The three sites can be distinguished by the numbers of surface tungsten atoms to which the hydrogen atom is coordinated (CN). Thus the $1 \mathrm{CN}$ site is on top of a tungsten atom, the $2 \mathrm{CN}$ site bridges two tungsten atoms, and the $5 \mathrm{CN}$ site is in the fourfold hole. The size of the surface array representing the $1 \mathrm{CN}$ and $5 \mathrm{CN}$ sites was increased to 21 tungsten atoms with an insignificant change in bonding energies. The presence of edges on these truncated surface arrays caused abnormal charge distribution, but these effects were reduced by adjusting the VOIP of the edge atoms. After this initial adjustment, there was no further adjustment of the tungsten orbitals' VOIP.

\section{RESULTS}

\section{A. Diatomic Molecules}

Energy-distance calculations were made for diatomic molecules to determine the parameters needed for the exponential repulsive energy term. These calculations also provided a basis for comparison with the chemisorbed system, in which the hydrogen was bonded to larger arrays of tungsten atoms. In Fig. 2, the bonding energy is plotted as a function of internuclear separation $r$ along the molecular axis $z$ for the $\mathrm{W}-\mathrm{W}$ and $\mathrm{W}-\mathrm{H}$ molecules. The bonding energy was calculated by subtracting from the total energy the energy of the ions of the $\mathrm{W}-\mathrm{H}$ molecule or the energy of the atoms of the W-W molecule at infinite separation. These arbitrary conventions compensate in part for the total neglect of Coulomb interactions in the EHMO method and have no influence on any conclusions drawn later.

Figures 2(a) and 2(b) display the clear need to include some of the inner orbitals of tungsten in the basis set in order to obtain a characteristic potential energy curve. The differences between curves $a$ and $b$ in Figs. $2(a)$ and $2(b)$ are the repulsive energies. In Fig. $3, \ln \Delta \epsilon$ is plotted as a function of internuclear separation $r$ for $\mathrm{W}-\mathrm{W}$ and $\mathrm{W}-\mathrm{H}$ molecules. From these plots, the values for the parameters $A$ and $\alpha$ needed to calculate the repulsive energy by the analytical exponential term were derived. Best values were found to be: $A_{\mathrm{WW}}=4096 . \mathrm{eV}, \alpha_{\mathrm{WW}}=0.298 \AA^{-1}, A_{\mathrm{WH}}=139 . \mathrm{eV}$, $\alpha_{\mathrm{WH}}=0.279 \AA^{-1}$.

As previously stated, the VOIP of the valence orbitals were adjusted as a function of atomic charge and iterated to self-consistency. For the diatomic molecule $\mathrm{W}_{2}$ the atomic charge on each tungsten atom was of course zero, for WH the charge on hydrogen was -0.10 and on tungsten +0.10 . Corresponding VOIP are implied by Table I. The molecular orbitals (LCAO coefficients) for

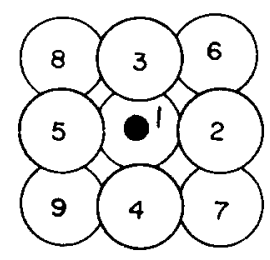

A) 5 CN SITE

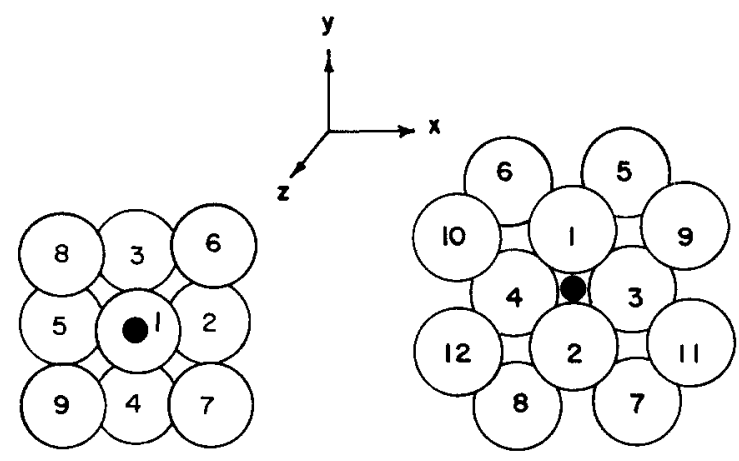

B) ICN SITE

C) 2 CN SITE
FIG. 1. Surface arrays used to model adsorption on different $W(100)$ surface sites. 


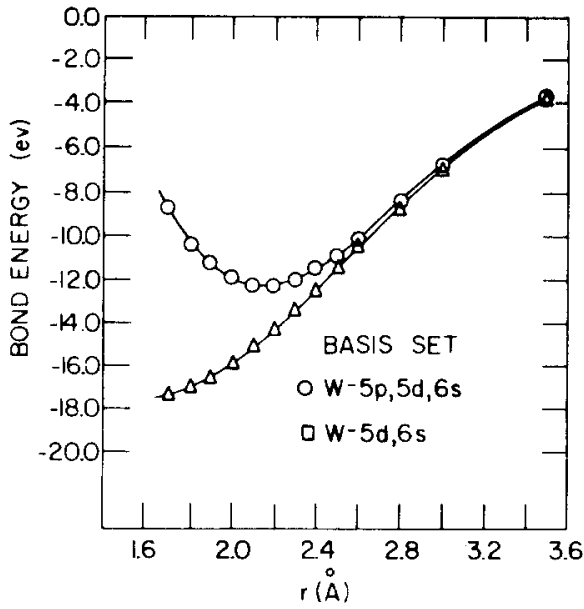

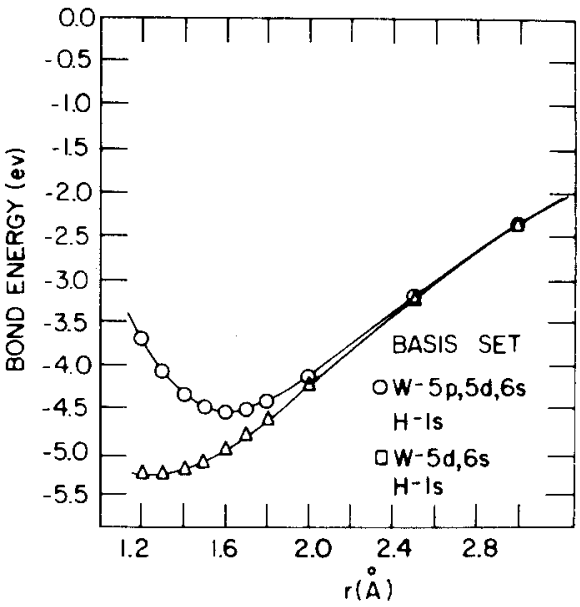

FIG. 2. Dependence of bond energy on bond distance for $\mathrm{W}_{2}$ (left figure) and WH (right figure) molecules with and without inclusion of W5 $p$ orbitals. the diatomic molecules at equilibrium separation are tabulated in Table II. Those for $\mathrm{W}-\mathrm{H}$ will prove useful in comparing the bonding of hydrogen to tungsten in the diatomic molecule with that in "surface molecules" such $\mathrm{W}_{9} \mathrm{H}$ used to model chemisorption.

Although the diatomic molecules in Table II are not known to exist, their EHMO bond lengths can be compared with the sum of the covalent radii of the atoms in the molecule. The Pauling single bond covalent radii of hydrogen and tungsten are 0.32 and $1.30 \AA$, respectively. The sum of the Pauling covalent radii for the diatomic tungsten molecule gives a bond length of $2.60 \AA$ for a single bond. The calculated bond length of $2.14 \AA$ implies a multiple bond whose order can be estimated from Pauling' s ${ }^{22}$ empirical rule,

$$
D(n)=D(1)-0.71 \log n
$$

Here $D(1)$ is the single bond length $(2.60 \AA), D(n)$ the observed bond length ( $2.14 \AA$ ), and $n$ the bond order. Pauling's rule leads to a value 4.4 for the bond order in reasonable agreement with the implications of Table II (five occupied bonding orbitals, one occupied antibonding orbital). Hence the calculated bond lengths appear to be reasonable and self-consistent.

A molecule of a hydrogen atom and two tungsten atoms was used to determine whether the repulsive energies as calculated in the analytical exponential term were pairwise additive. In this molecule, the tungsten atoms were separated by $3.16 \AA$, a characteristic distance on a tungsten (100) surface, and the hydrogen was equidistant from these two tung-

TABLE II. Occupied molecular orbitals for the diatomic molecules, W-W and $\mathrm{W}-\mathrm{H}$.

\begin{tabular}{|c|c|c|c|c|c|c|}
\hline $\begin{array}{c}\text { W-W } \\
\text { Energy }(e V)\end{array}$ & $\begin{array}{c}\Psi_{1} \\
(-10.63)\end{array}$ & $\begin{array}{c}\Psi_{2} \\
(-10.57)\end{array}$ & $\begin{array}{c}\boldsymbol{\Psi}_{3} \\
(-10.57)\end{array}$ & $\begin{array}{c}\boldsymbol{\Psi}_{4} \\
(-9.57)\end{array}$ & $\begin{array}{c}\boldsymbol{\Psi}_{5} \\
(-9.57)\end{array}$ & $\begin{array}{c}\Psi_{6} \\
(-9.23)\end{array}$ \\
\hline \multicolumn{7}{|l|}{$W(1,2)$} \\
\hline $5 d_{z} 2$ & -0.16 & & & \multirow{6}{*}{0.68} & \multirow{6}{*}{0.68} & -0.63 \\
\hline $5 d_{x z}$ & & \pm 0.62 & & & & \\
\hline $5 d_{x^{2}-y^{2}}$ & & & & & & \\
\hline $5 d_{\mathrm{yz}}$ & & & \pm 0.62 & & & \\
\hline $5 d_{x y}$ & & & & & & \\
\hline $6 s$ & 0.52 & & & & & -0.16 \\
\hline $\begin{array}{c}\text { W-H } \\
\text { Energy }(\mathrm{eV})\end{array}$ & $\begin{array}{c}\Psi_{1} \\
(-12.23)\end{array}$ & $\begin{array}{c}\Psi_{2} \\
(-9.42)\end{array}$ & $\begin{array}{c}\boldsymbol{\Psi}_{3} \\
(-9.42)\end{array}$ & $\begin{array}{c}\Psi_{4} \\
(-9.42)\end{array}$ & $\begin{array}{c}\Psi_{5} \\
(-9.42)\end{array}$ & \\
\hline \multicolumn{7}{|l|}{$\mathrm{H}$} \\
\hline $1 s$ & -0.62 & & & & & \\
\hline \multicolumn{7}{|l|}{$\mathrm{W}$} \\
\hline $5 d_{z^{2}}$ & -0.47 & & & \multirow{6}{*}{1.00} & \multirow{6}{*}{1.00} & \\
\hline $5 d_{x z}$ & & 1.00 & & & & \\
\hline $5 d_{x^{2}-y^{2}}$ & & & 1.00 & & & \\
\hline $5 d_{y z}$ & & & & & & \\
\hline $5 d_{x y}$ & & & & & & \\
\hline $6 s$ & -0.26 & & & & & \\
\hline
\end{tabular}




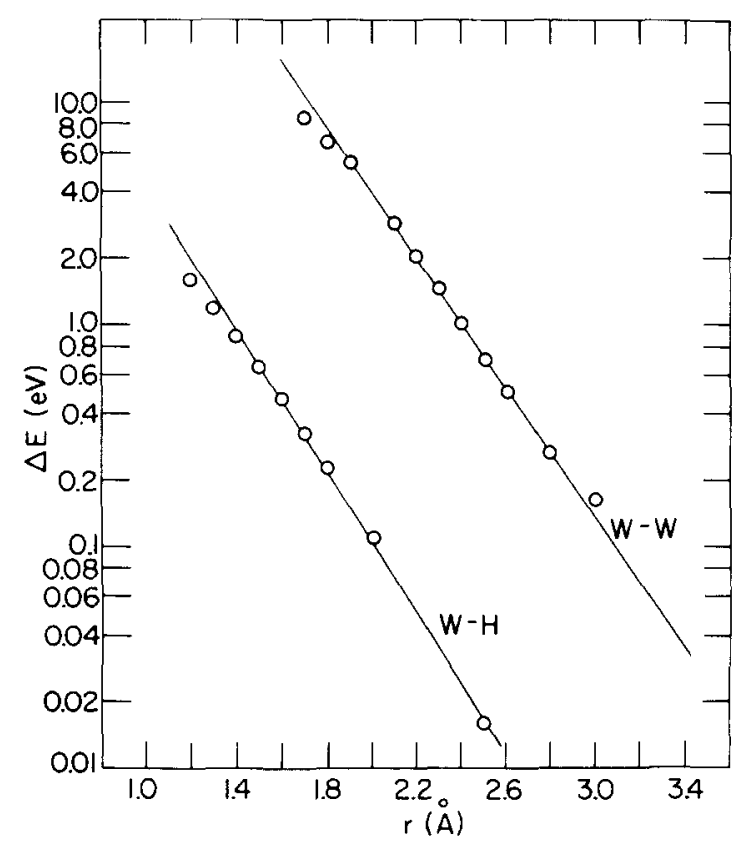

FIG. 3. Dependence of nonbonding (5p orbital) repulsive energy on distance for $\mathrm{W}_{2}$ and $\mathrm{WH}$ molecules.

sten atoms. The repulsive energies plotted as curve $a$ in Fig. 4 are determined by the differences between the energies calculated by the EHMO technique where the basis set of atomic orbitals included the $5 p$ as well as the valence $(6 s, 5 d)$ orbitals of the tungsten atoms and those calculated where the $5 p$ orbitals were omitted from the basis set. The repulsive energies, as calculated by the analytical exponential repulsive term for two tungsten-hydrogen interactions, are plotted as curve $b$ in Fig. 4. From the comparison of curves $a$ and $b$, it is confirmed that the repulsive energy is reasonably well represented by the sum of all pairwise interactions.

\section{B. Hydrogen Chemisorbed on a W(100) Surface}

Before calculations were performed with the hydrogen at the proposed chemisorbed sites, it was necessary to make adjustments on the VOIP of the edge tungsten atoms. Because the edge atoms of the surface arrays were missing nearest and next-nearest neighbors, their VOIP were adjusted to prevent abnormal charge distribution. The VOIP of the $5 d$ and $6 s$ orbitals of the four corner atoms of the arrays in Figs. 1(a) and 1(b) were adjusted to -8.52 and $-7.52 \mathrm{eV}$, respectively; these same orbitals on the other four edge atoms were adjusted to -8.76 and $-7.76 \mathrm{eV}$, respectively. The orbitals of the eight edge atoms of the array in Fig. 1c were adjusted to $-8.68 \mathrm{eV}$ for the $5 d$ orbitals and $-7.68 \mathrm{eV}$ for the $6 \mathrm{~s}$ orbital. The VOIP of the remaining atoms in these arrays were not adjusted.

Mattheiss' ${ }^{23}$ band theory calculation on tungsten provides two check points for our EHMO calculations on the $\mathrm{W}_{9}$ molecule. First, he finds a $d$ bandwidth of $10.47 \mathrm{eV}$. We take as the EHMO analog the difference in energies between highest and lowest molecular orbitals having $d$ character on atom 1 in the surface array of Fig. 1(a). The lowest energy of such an orbital is $\Psi_{2}$ [shown in Fig. 6(a)], the highest (not shown because it is unoccupied) is $9.45 \mathrm{eV}$ higher. Second, Mattheiss finds that the bottom of the $d$ band lies $1.36 \mathrm{eV}$ above the bottom of the conduction band. Our analog is the difference between $\Psi_{1}$ in Fig. 6(a) and the first occupied orbital of $d$ character [not totally symmetric and therefore not shown in Fig. 6(a)]; this difference is $1.34 \mathrm{eV}$, remarkably close to Mattheiss' result.

Calculations were then carried out with hydrogen bonded at the three possible symmetric sites. The minimum charge transfer to the hydrogen occurred with hydrogen bonded at the $5 \mathrm{CN}$ site and the maximum with the hydrogen bonded at the 1 $\mathrm{CN}$ site. In the initial calculations, the VOIP of the hydrogen 1s orbital was adjusted as a function of atomic charge $q$ and iterated to self-consistency holding the tungsten orbital VOIP constant at values previously given. The final iteration VOIP were $-11.34 \mathrm{eV}$ at the $5 \mathrm{CN}$ site, $-9.54 \mathrm{eV}$ at the $2 \mathrm{CN}$ site, and $-8.84 \mathrm{eV}$ at the $1 \mathrm{CN}$ site. These differences raised the possibility that energy differences between various absorption configurations might reflect too strongly the different parametrizations of the hydrogen $1 s$ VOIP. Since these energy differences were of principle concern, the calculations were repeated using a VOIP of -10.30 for all sites. Comparing the two sets of calculations, it was found that differences in bond energy and equilibrium distances between configurations

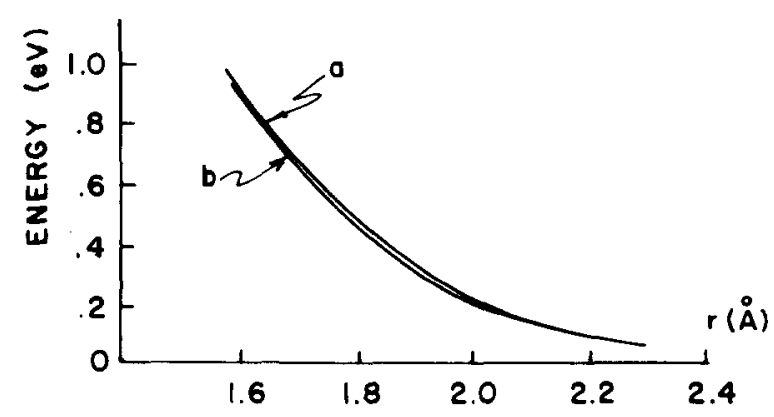

FIG. 4. Additivity of nonbonded orbital repulsive energy. (a) Difference in $E(r)$ for $\mathrm{W}_{2} \mathrm{H}$ between calculations including and omitting $W 5 p$ orbitals. (b) $E(r)$ calculated by pairwise addition of analytic repulsive energy functions. 


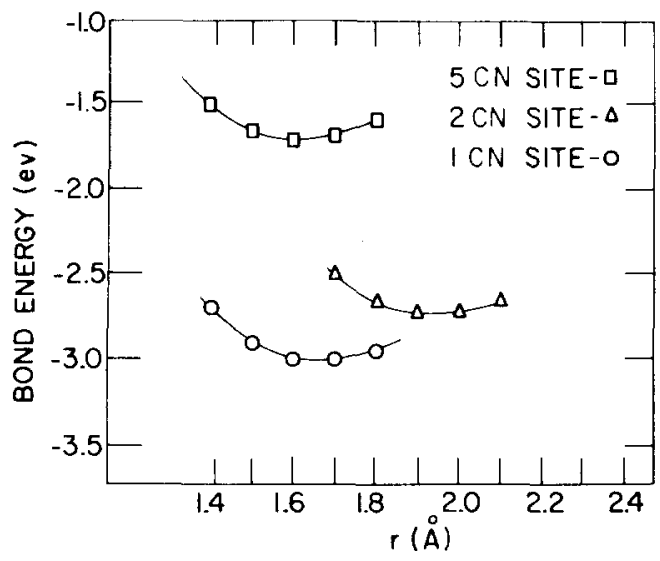

FIG. 5. Bond energies as functions of distances for hydrogen adsorbed on different $W(100)$ surface sites.

were almost unaffected, and most importantly that the order of bonding energies among the three configurations remained unchanged. Hence use of a fixed hydrogen VOIP of -10 . $\mathrm{eV}$ was adopted for all subsequent calculations for computational simplicity.

The dependence of bonding energy $E$ on internuclear separation $r$ is plotted in Fig. 5 for hydrogen bonded at each of the three symmetric sites. The internuclear separation was the distance of hydrogen to the nearest tungsten atom(s) in the surface atom arrays. In the case of the $5 \mathrm{CN}$ site, it was the distance to the tungsten atom in the hole, for the $2 \mathrm{CN}$ site, it was the distance to the two surface atoms, and for the $1 \mathrm{CN}$ site, it was the distance to the single tungsten atom. The bond energy $E$ was calculated according to the following relationship:

$$
E=\sum_{\mathrm{w}_{n} \mathrm{H}} n_{i} \epsilon_{i}-\sum_{\mathrm{w}_{n}} n_{i} \epsilon_{i}-\epsilon_{\mathrm{H}}+\sum_{\mathrm{w}_{n} \mathrm{H}} A e^{-r / \alpha},
$$

where the first term is the summation over the occupied molecular orbitals of energy $\epsilon_{i}$ of the surface "molecule" $\mathrm{W}_{n} \mathrm{H}$, the next term is the summation over the occupied molecular orbitals of energies $\epsilon_{i}$ of the $\mathrm{W}_{n}$ part of the "molecule" separated from the hydrogen, and the next term is the energy of the singly occupied atomic orbital of a separated hydrogen atom. The last term, the repulsive energy term, represents the repulsion of the occupied inner nonbonding orbitals (omitted from initial calculations), and the summation extends over all hydrogen-tungsten pairs in the surface array. This term is not necessary to determine the bond energy if the inner orbitals are included in the basis set for the MO calculations. Table III presents bonding energies, equilibrium distances, and charges corresponding to the energy minima in Fig. 5.
A somewhat simpler description and a better understanding of the bonding between hydrogen and the surface arrays can be obtained by using group theory. First, the surface "molecules" must be assigned to a symmetry group. The $1 \mathrm{CN}$ and 5 $\mathrm{CN}$ sites on the $\mathrm{W}(100)$ surface have fourfold symmetry and the surface arrays in Fig. 1 representing the surface for each of these symmetric sites belong to the $C_{4 v}$ symmetry group. The $2 \mathrm{CN}$ site has twofold symmetry and the surface array representing this site belongs to the $C_{2 v}$ symmetry group. Because the hydrogen $1 s$ orbital is totally symmetric and belongs to the $a_{1}$ irreducible representation in these symmetry groups, it will interact only with the molecular orbitals of the tungsten arrays belonging to this same totally symmetric irreducible representation $a_{1}$. For the $C_{4 v}$ surface arrays with nine tungsten atoms [Figs. 1(a) and 1(b)], there are 12 totally symmetric molecular orbitals formed by the valence orbitals of which only 5 are occupied. The $C_{2 v}$ surface array [Fig. 1(c)] with 12 tungsten atoms has 20 totally symmetric molecular orbitals of which 11 are occupied. An additional totally symmetric molecular orbital is formed as the hydrogen $1 \mathrm{~s}$ orbital interacts with molecular orbitals of the $\mathrm{W}_{n}$ arrays.

Molecular orbital energy diagrams are shown in Fig. 6 for the hydrogen interacting with each of the surface arrays at equilibrium separation. The totally symmetric molecular orbitals of the tungsten arrays are indicated on the left side of the panels in Fig. 6 with the hydrogen $1 \mathrm{~s}$ orbital on the right side and in the middle are the molecular orbitals formed by the $\mathrm{W}_{n} \mathrm{H}$ surface "molecule." The lines joining the molecular orbitals of the separated $\mathrm{W}_{n}$ and $\mathrm{H}$ atomic orbital with those of the combined $\mathrm{W}_{n} \mathrm{H}$ show the change in energy of the $\mathrm{W}_{n}$ molecular orbitals as they interact with the hydrogen in qualitative form; corresponding quantitative information can be obtained by comparing Tables IV and V (which tabulate the occupied totally symmetric molecular orbitals of the $\mathrm{W}_{9}$ and $\mathrm{W}_{12}$ surface arrays) with Tables VI-VIII (which tabulate the totally symmetric orbitals of the $\mathrm{W}_{n} \mathrm{H}$ surface "molecules"). From these tables, it is observed that hydrogen makes only a small contribution to

TABLE III. Energies, equilibrium distances, and charges for hydrogen bonded to different $W(100)$ surface sites.

\begin{tabular}{lccc}
\hline \hline Site & $\begin{array}{c}\text { Bond energy } \\
(\mathrm{eV})\end{array}$ & $\begin{array}{c}r_{e} \\
(\AA)\end{array}$ & $\begin{array}{c}\text { Charge } \\
|e|\end{array}$ \\
\hline $1 \mathrm{CN}$ & 3.00 & 1.65 & -0.36 \\
$2 \mathrm{CN}$ & 2.74 & 1.96 & -0.26 \\
$5 \mathrm{CN}$ & 1.72 & 1.61 & +0.04 \\
\hline
\end{tabular}


a) ICN SITE

b) $2 \mathrm{CN}$ SITE

c) $5 \mathrm{CN}$ SITE

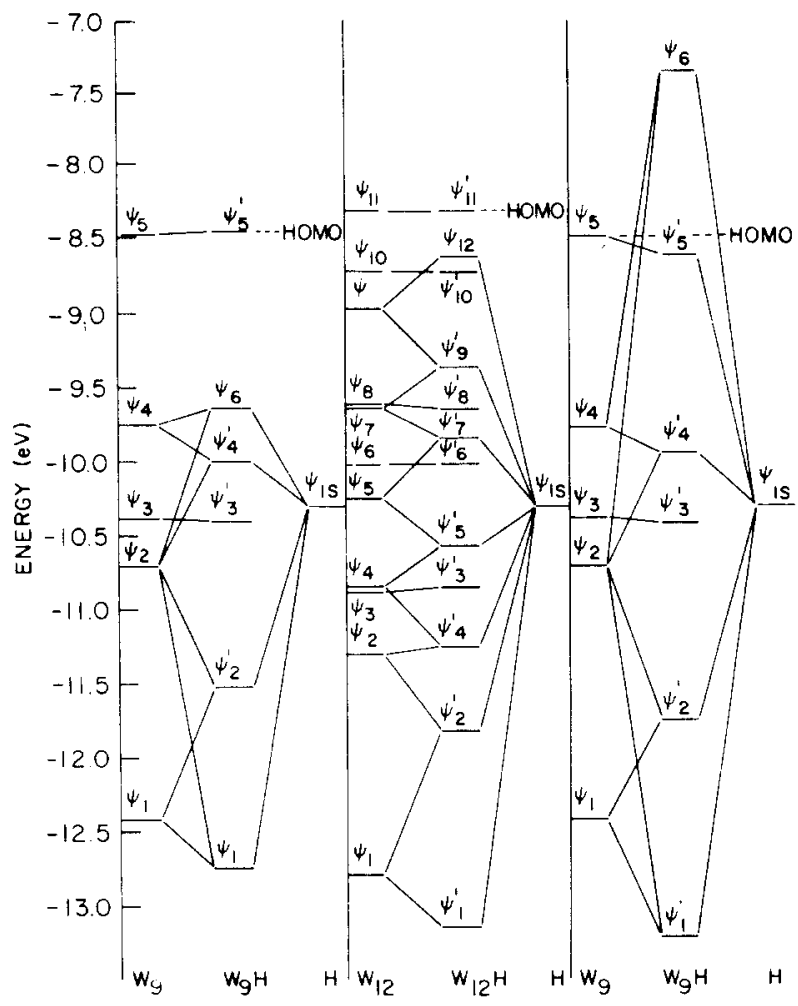

FIG. 6. Molecular orbital-energy diagrams for the totally symmetric occupied orbitals for hydrogen adsorbed on different W (100) surface sites.

some of the molecular orbitals of the surface "molecule." These are indicated in Fig. 6 as orbitals not joined by a line to the hydrogen 1 s orbital.
It is interesting to note that, for the $1 \mathrm{CN}$ and $2 \mathrm{CN}$ sites, interaction of the hydrogen atom with the $k$ occupied molecular orbitals in the totally symmetric $a_{1}$ representation leads to $k+1$ molecular orbitals all of which are below the highest occupied molecular orbital (HOMO), and which are correspondingly occupied and contribute to bonding in the $\mathrm{W}_{n} \mathrm{H}$ molecule. In the $5 \mathrm{CN}$ site one of the molecular orbitals in the $\mathrm{W}_{n} \mathrm{H}$ molecule is above the HOMO $\left[\Psi_{6}\right.$ in Fig. 6(c) $]$ and so does not contribute to bonding.

\section{DISCUSSION}

The observed ${ }^{6,9}$ LEED patterns from a $C(2 \times 2)$ surface structure of hydrogen on the $W(100)$ surface indicate that hydrogen must be chemisorbed at a site with four-fold symmetry, either the $5 \mathrm{CN}$ or $1 \mathrm{CN}$ site. Evidence for this comes from the diffraction spots of the $C(2 \times 2)$ surface structure, where the spots at the half-order position $\left[\left(\frac{1}{2}, \frac{1}{2}\right)\right.$, $\left(\frac{1}{2}, \frac{3}{2}\right)$, etc. ] are diffuse, resulting from $C(2 \times 2)$ domains on the surface which are not in phase. The facts that the spots at the integral order positions $[(1,0),(1,1)$, etc. $]$ are sharp and only those at the half-order positions are diffuse indicate that hydrogen must be chemisorbed at sites with fourfold symmetry in the $C(2 \times 2)$ surface structure. ${ }^{24}$ From the results of our calculations as shown in Table III, the $1 \mathrm{CN}$ site can be chosen as the preferred site with fourfold symmetry for the chemisorbed hydrogen atom.

Early experimental evidence led to conflicting models for hydrogen chemisorbed on a $\mathrm{W}(100)$ surface including: atoms bonded at a single site, ${ }^{6,8}$ atoms bonded at different sites, ${ }^{9}$ and a mixture of

TABLE IV. Totally symmetric molecular orbitals for the surface arrays representing the $1 \mathrm{CN}$ and $5 \mathrm{CN}$ sites.

\begin{tabular}{|c|c|c|c|c|c|}
\hline $\begin{array}{l}\text { Molecular orbital } \\
\text { Energy (eV) }\end{array}$ & $\begin{array}{c}\Psi_{1} \\
(-12.43)\end{array}$ & $\begin{array}{c}\Psi_{2} \\
(-10.69)\end{array}$ & $\begin{array}{c}\Psi_{3} \\
(-10.38)\end{array}$ & $\begin{array}{c}\Psi_{4} \\
(-9.77) \\
\end{array}$ & $\begin{array}{c}\Psi_{5} \\
(-8.47) \\
\end{array}$ \\
\hline \multicolumn{6}{|l|}{$\mathrm{W}(1)$} \\
\hline $5 d_{z^{2}}$ & & -0.54 & & -0.10 & -0.19 \\
\hline $6 s$ & -0.24 & & & & \\
\hline \multicolumn{6}{|l|}{$W(2,3,4,5)$} \\
\hline $5 d z$ & & 0.22 & 0.17 & 0.16 & \\
\hline $5 d_{x z}(2,5)$ & & \pm 0.15 & & \pm 0.31 & \pm 0.07 \\
\hline $5 d_{x^{2}-y^{2}}$ & & \pm 0.12 & \pm 0.30 & \pm 0.19 & \pm 0.05 \\
\hline $5 d_{y z}(3,4)$ & & \pm 0.15 & & \pm 0.31 & \pm 0.07 \\
\hline $6 s$ & -0.18 & & 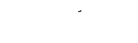 & & \\
\hline \multicolumn{6}{|l|}{$W(6,7,8,9)$} \\
\hline $5 d_{z^{2}}$ & & -0.10 & -0.19 & & 0.26 \\
\hline $5 d_{x z}$ & & & \pm 0.11 & & \pm 0.28 \\
\hline $5 d_{y z}$ & & & \pm 0.11 & & \pm 0.28 \\
\hline $5 d_{x y}$ & & \pm 0.12 & & \pm 0.22 & \pm 0.10 \\
\hline $6 s$ & -0.12 & & & & \\
\hline
\end{tabular}


TABLE V. Totally symmetric molecular orbitals for the surface array representing the $2 \mathrm{CN}$ site.

\begin{tabular}{|c|c|c|c|c|c|c|c|c|c|c|c|}
\hline & $\begin{array}{c}\psi_{1} \\
(-12.78) \\
\end{array}$ & $\begin{array}{c}\psi_{2} \\
(-11.31) \\
\end{array}$ & $\begin{array}{c}\psi_{3} \\
(-10.87) \\
\end{array}$ & $\begin{array}{c}\psi_{4} \\
(-10.82) \\
\end{array}$ & $\begin{array}{c}\psi_{5} \\
(-10.25) \\
\end{array}$ & $\begin{array}{c}\psi_{6} \\
(-10.03) \\
\end{array}$ & $\begin{array}{c}\psi_{7} \\
(-9.68) \\
\end{array}$ & $\begin{array}{c}\psi_{8} \\
(-9.62) \\
\end{array}$ & $\begin{array}{c}\psi_{9} \\
(-8.97) \\
\end{array}$ & $\begin{array}{c}\psi_{10} \\
(-8.72) \\
\end{array}$ & $\begin{array}{c}\psi_{11} \\
(-8.32) \\
\end{array}$ \\
\hline \multicolumn{12}{|l|}{$W(1,2)$} \\
\hline $\begin{array}{l}5 d_{z^{2}} \\
5 d_{x}{ }^{2}-y^{2} \\
5 d_{y z} \\
6 s\end{array}$ & 0.18 & $\begin{array}{r}0.33 \\
\pm 0.12\end{array}$ & $\begin{array}{l}-0.36 \\
\pm 0.08\end{array}$ & $\begin{array}{l}-0.09 \\
\pm 0.32\end{array}$ & $\begin{array}{l}-0.19 \\
\pm 0.21\end{array}$ & $\begin{array}{l}-0.08 \\
-0.06\end{array}$ & $\begin{array}{r}0.09 \\
-0.05 \\
\pm 0.17\end{array}$ & $\begin{array}{r}-0.15 \\
-0.08 \\
\pm 0.18\end{array}$ & $\begin{array}{l}-0.30 \\
\pm 0.10\end{array}$ & $\begin{array}{l}-0.14 \\
\pm 0.12\end{array}$ & $\begin{array}{l}0.15 \\
0.08\end{array}$ \\
\hline \multicolumn{12}{|l|}{$\mathrm{W}(3,4)$} \\
\hline $\begin{array}{l}5 d_{x^{2}} \\
5 d_{x z} \\
5 d_{x^{2}-y^{2}} \\
6 s\end{array}$ & 0.18 & $\begin{array}{r} \pm 0.12 \\
-0.33\end{array}$ & $\begin{array}{r}0.36 \\
\pm 0.08\end{array}$ & $\begin{array}{r}-0.09 \\
\pm 0.32\end{array}$ & $\begin{array}{r} \pm 0.21 \\
-0.19\end{array}$ & $\begin{array}{r}-0.08 \\
0.05\end{array}$ & $\begin{array}{r}-0.09 \\
\pm 0.17 \\
0.05\end{array}$ & $\begin{array}{r}-0.15 \\
\pm 0.18 \\
0.08\end{array}$ & $\begin{array}{r} \pm 0.10 \\
-0.30\end{array}$ & $\begin{array}{r} \pm 0.12 \\
0.14\end{array}$ & $\begin{array}{r}-0.15 \\
0.08\end{array}$ \\
\hline \multicolumn{12}{|c|}{$\mathrm{W}(5,6,7,8)$} \\
\hline $\begin{array}{l}5 d_{z^{2}} \\
5 d_{x z} \\
5 d_{x^{2}-y^{2}} \\
5 d_{y z} \\
5 d_{x y} \\
6 s\end{array}$ & 0.10 & -0.13 & $\begin{array}{r}0.12 \\
\pm 0.05 \\
0.05\end{array}$ & $\begin{array}{r}0.05 \\
\pm 0.07 \\
-0.11 \\
\pm 0.12\end{array}$ & $\begin{array}{r}-0.17 \\
\pm 0.14 \\
0.10\end{array}$ & $\begin{array}{r}0.07 \\
\pm 0.23 \\
-0.16 \\
\pm 0.07 \\
\pm 0.08\end{array}$ & $\begin{array}{r}0.08 \\
\\
0.05 \\
\pm 0.13 \\
\pm 0.24\end{array}$ & $\begin{array}{r}0.06 \\
\pm 0.09 \\
\pm 0.19 \\
\pm 0.18\end{array}$ & $\begin{array}{r}0.08 \\
-0.20 \\
\pm 0.14 \\
\pm 0.09\end{array}$ & $\begin{array}{l}-0.12 \\
-0.05 \\
\pm 0.24 \\
\pm 0.18\end{array}$ & $\begin{array}{r}0.11 \\
\pm 0.26 \\
-0.14 \\
\pm 0.10 \\
\pm 0.08\end{array}$ \\
\hline \multicolumn{12}{|c|}{$\mathrm{W}(9,10,11,12)$} \\
\hline $\begin{array}{l}5 d_{z^{2}} \\
5 d_{x z} \\
5 d_{x^{2}-y^{2}} \\
5 d_{y z} \\
5 d_{x y} \\
6 s\end{array}$ & 0.10 & 0.13 & $\begin{array}{l} \pm 0.05 \\
\pm 0.05\end{array}$ & $\begin{array}{r}0.11 \\
\pm 0.07 \\
\pm 0.12\end{array}$ & $\begin{array}{r}0.17 \\
\\
0.10 \\
\pm 0.14\end{array}$ & $\begin{array}{r}0.07 \\
\pm 0.07 \\
0.16 \\
\pm 0.23 \\
\pm 0.08\end{array}$ & $\begin{array}{r}0.08 \\
\pm 0.13 \\
0.05 \\
\pm 0.24\end{array}$ & $\begin{array}{r}0.06 \\
\pm 0.19 \\
\pm 0.09 \\
\pm 0.18\end{array}$ & $\begin{array}{r}0.08 \\
\pm 0.14 \\
\pm 0.20 \\
\pm 0.09\end{array}$ & $\begin{array}{r}-0.12 \\
\pm 0.24 \\
0.05 \\
\pm 0.18\end{array}$ & $\begin{array}{c}-0.11 \\
\pm 0.10 \\
-0.14 \\
\pm 0.26 \\
\pm 0.08\end{array}$ \\
\hline
\end{tabular}


TABLE VI. Totally symmetric molecular orbitals for the $\mathrm{W}_{9} \mathrm{H}$ surface "molecule" where hydrogen is bonded at a $1 \mathrm{CN}$ site.

\begin{tabular}{|c|c|c|c|c|c|c|}
\hline $\begin{array}{l}\text { Molecular orbital } \\
\text { energy (eV) }\end{array}$ & $\begin{array}{c}\boldsymbol{\Psi}_{1}^{\prime} \\
(-12.74)\end{array}$ & $\begin{array}{c}\Psi_{2}^{\prime} \\
(-11.52)\end{array}$ & $\begin{array}{c}\Psi_{3}^{\prime} \\
(-10.39) \\
\end{array}$ & $\begin{array}{c}\Psi_{4}^{\prime} \\
(-10.01) \\
\end{array}$ & $\begin{array}{c}\boldsymbol{\Psi}_{6} \\
(-9.66) \\
\end{array}$ & $\begin{array}{c}\Psi_{5}^{\prime} \\
(-8.46) \\
\end{array}$ \\
\hline \multicolumn{7}{|l|}{$\mathrm{H}$} \\
\hline $1 s$ & 0.36 & -0.49 & -0.06 & 0.36 & 0.26 & -0.05 \\
\hline \multicolumn{7}{|l|}{$W(1)$} \\
\hline $\begin{array}{l}5 d_{z^{2}} \\
6 s\end{array}$ & $\begin{array}{l}0.16 \\
0.24\end{array}$ & $\begin{array}{r}-0.52 \\
0.06\end{array}$ & & $\begin{array}{r}-0.06 \\
0.05\end{array}$ & & 0.12 \\
\hline \multicolumn{7}{|l|}{$W(2,3,4,5)$} \\
\hline $\begin{array}{l}5 d_{z^{2}} \\
5 d_{x z}(2,5) \\
5 d_{x^{2}-y^{2}} \\
5 d_{y z}(3,4) \\
6 s\end{array}$ & 0.13 & $\begin{array}{r}0.07 \\
\pm 0.05 \\
\pm 0.06 \\
\pm 0.05 \\
0.11\end{array}$ & $\begin{array}{l}-0.22 \\
\pm 0.27\end{array}$ & $\begin{array}{r}0.11 \\
\pm 0.30 \\
\pm 0.10 \\
\pm 0.30 \\
-0.08\end{array}$ & $\begin{array}{r}0.26 \\
\pm 0.19 \\
\pm 0.27 \\
\pm 0.19 \\
-0.05\end{array}$ & $\begin{array}{l} \pm 0.06 \\
\pm 0.08 \\
\pm 0.06\end{array}$ \\
\hline \multicolumn{7}{|l|}{$W(6,7,8,9)$} \\
\hline $\begin{array}{l}5 d_{z^{2}} \\
5 d_{x z} \\
5 d_{y z} \\
5 d_{x y} \\
6 s\end{array}$ & 0.10 & $\begin{array}{r} \pm 0.04 \\
0.07\end{array}$ & $\begin{array}{r}0.21 \\
\pm 0.11 \\
\pm 0.11\end{array}$ & \pm 0.22 & -0.07 & $\begin{array}{l}-0.26 \\
\pm 0.28 \\
\pm 0.28 \\
\pm 0.11\end{array}$ \\
\hline
\end{tabular}

atomic and molecular species bonded to the surface. ${ }^{7}$ Recent evidence ${ }^{10,11}$ indicates that atomic species are bonded at one kind of surface site; the two peaks, $\beta_{1}$ and $\beta_{2}$, in the flash desorption spectrum are a result of a density-dependent interaction on the surface. The high-temperature state, $\beta_{2}$, has been associated with a $C(2 \times 2)$ surface structure and as the remaining sites are populated, a $(1 \times 1)$ surface structure results. From the results of our calculating, these sites must be the $1 \mathrm{CN}$ sites on the $\mathrm{W}(100)$ surface.

In the WH molecule, the hydrogen $1 s$ orbital appears only in the single molecular orbital $\Psi_{1}$ (see Table III). In this case the tungsten-hydrogen bond can be interpreted as a simple single bond involving a hydrogen $1 s$ orbital and a tungsten $s d$ hybrid orbital. It is tempting to interpret the $1 \mathrm{CN}$ preference for hydrogen adsorption as reflecting a preference for simple single bonding to the tungsten atom below it. The present treatment is not well designed to provide a justification for such an interpretation. Table VI shows that the molecular orbitals $\Psi_{1}^{\prime}, \Psi_{2}^{\prime}, \Psi_{4}^{\prime}$, and $\Psi_{6}$ all have substantial hydrogen 1 s contributions; while the principal tungsten orbital contributions to $\Psi_{1}^{\prime}$ and $\Psi_{2}^{\prime}$ arise from the tungsten atom underlying the hydrogen atom this atom contributes almost negligibly to $\Psi_{4}^{\prime}$ and $\Psi_{6}$, whose tungsten contributions come largely from the $d$ orbitals of the four tungsten atoms surrounding the adsorption site. Such molecular orbitals are not well suited for interpretation in terms of localized bonding; for this purpose optimally localized orbitals are more appropriate as shown by Ruedenberg and Edmiston. ${ }^{25}$ The tungsten atoms which are involved with the bonding of the hydrogen atom retain their bonds with the neighboring tungsten atoms, but their strengths change as a result of the chemisorbed hydrogen. The reduced overlap population calculated by the Mulliken ${ }^{19}$ method can assess the relative change in strengths of these tungstentungsten bonds. With hydrogen bonded at the 1 $\mathrm{CN}$ site, the reduced overlap population of the central tungsten atom with each of its four nearestneighboring tungsten atoms is reduced $15 \%$.

The small difference in the bonding energy between a $1 \mathrm{CN}$ site and a $2 \mathrm{CN}$ site can account for the observed mobility of hydrogen on tungsten surfaces. Gomer et al. ${ }^{26}$ observed the diffusion of hydrogen on a tungsten surface at a temperature of $180^{\circ} \mathrm{K}$ and reported the activation energy for diffusion $E_{d}$ was generally $10 \%-20 \%$ of the bonding energy $E_{a}$, that both $E_{a}$ and $E_{d} / E_{a}$ were lowest on most closely packed faces, and that the lowest value of $E_{d}$ observed was $5.9 \mathrm{kcal}(0.26 \mathrm{eV})$. If the diffusion of hydrogen on the $\mathrm{W}(100)$ surface occurs from one $1 \mathrm{CN}$ site to another $1 \mathrm{CN}$ site via a 2 CN site, and the only energy barrier to diffusion is the difference between bonding energies at these two sites, then the activation energy for diffusion would be $0.25 \mathrm{eV}$. 
TABLE VII. Totally symmetric molecular orbitals for the $\mathrm{W}_{12} \mathrm{H}$ surface "molecule" where hydrogen is bonded at a 2 CN site.

\begin{tabular}{|c|c|c|c|c|c|c|c|c|c|c|c|c|}
\hline $\begin{array}{c}\text { Molecular } \\
\text { orbital } \\
\text { energy }(\mathrm{eV})\end{array}$ & $\begin{array}{c}\Psi_{1}^{\prime} \\
(-13,15)\end{array}$ & $\begin{array}{c}\Psi_{2}^{\prime} \\
(-11.82)\end{array}$ & $\begin{array}{c}\Psi_{4}^{\prime} \\
(-11.25)\end{array}$ & $\begin{array}{c}\Psi_{3}^{\prime} \\
(-10.85)\end{array}$ & $\begin{array}{c}\boldsymbol{\Psi}_{5}^{\prime} \\
(-10.57)\end{array}$ & $\begin{array}{c}\boldsymbol{\Psi}_{6}^{\prime} \\
(-10.03)\end{array}$ & $\begin{array}{c}\Psi_{?}^{\prime} \\
(-9.84)\end{array}$ & $\begin{array}{c}\dot{\psi}_{8}^{\prime} \\
(-9.65)\end{array}$ & $\begin{array}{c}\boldsymbol{\psi}_{\dot{g}}^{\prime} \\
(-9.38)\end{array}$ & $\begin{array}{c}\dot{\Psi}_{10}^{\prime} \\
(-8.73)\end{array}$ & $\begin{array}{c}\Psi_{12} \\
(-8.62) \\
\end{array}$ & $\begin{array}{c}\Psi_{11}^{\prime} \\
(-8.32)\end{array}$ \\
\hline \multicolumn{13}{|l|}{$\mathrm{H}$} \\
\hline $1 s$ & 0.35 & -0.44 & 0.13 & -0.02 & -0.16 & -0.02 & 0.18 & -0.04 & -0.22 & 0.06 & -0.21 & 0.02 \\
\hline \multicolumn{13}{|l|}{$\mathrm{W}(1,2)$} \\
\hline $\begin{array}{l}5 d_{z^{2}} \\
5 d_{x^{2}-y^{2}} \\
5 d_{y z z} \\
6 s\end{array}$ & $\begin{array}{r}-0.07 \\
\pm 0.09 \\
0.18\end{array}$ & $\begin{array}{r}0.05 \\
0.26 \\
\pm 0.24\end{array}$ & $\begin{array}{r}0.24 \\
\pm 0.27\end{array}$ & $\begin{array}{l}-0.36 \\
\pm 0.13\end{array}$ & $\begin{array}{r}-0.09 \\
0.09\end{array}$ & 0.08 & \pm 0.07 & $\begin{array}{l}0.16 \\
0.10\end{array}$ & $\begin{array}{l}-0.09 \\
-0.10 \\
\pm 0.14\end{array}$ & $\begin{array}{l}-0.05 \\
\pm 0.13\end{array}$ & $\begin{array}{l}-0.19 \\
-0.05\end{array}$ & $\begin{array}{l}0.15 \\
0.08\end{array}$ \\
\hline \multicolumn{13}{|l|}{$W(3,4)$} \\
\hline $\begin{array}{l}5 d_{z^{2}} \\
5 d_{x z} \\
5 d_{x^{2}-y^{2}} \\
6 s\end{array}$ & 0.15 & $\begin{array}{r}-0.09 \\
-0.08 \\
0.10\end{array}$ & $\begin{array}{r}0.11 \\
\pm 0.14 \\
-0.32\end{array}$ & $\begin{array}{r}0.25 \\
\pm 0.23\end{array}$ & $\begin{array}{r}0.22 \\
\pm 0.31 \\
0.15\end{array}$ & $\begin{array}{r}0.09 \\
-0.07\end{array}$ & $\begin{array}{r}-0.16 \\
0.11\end{array}$ & $\begin{array}{r}0.06 \\
\pm 0.25\end{array}$ & $\begin{array}{r}-0.07 \\
\pm 0.04 \\
0.13\end{array}$ & $\begin{array}{r} \pm 0.07 \\
0.24\end{array}$ & $\begin{array}{r} \pm 0.16 \\
-0.26\end{array}$ & -0.15 \\
\hline \multicolumn{13}{|l|}{$W(5,6,7,8)$} \\
\hline $\begin{array}{l}5 d_{z^{2}} \\
5 d_{x z} \\
5 d_{x^{2}-y^{2}} \\
5 d_{y z} \\
5 d_{x y} \\
6 s\end{array}$ & 0.06 & $\begin{array}{l} \pm 0.05 \\
-0.08\end{array}$ & $\begin{array}{l}-0.08 \\
\pm 0.06 \\
\pm 0.03\end{array}$ & $\begin{array}{r}0.13 \\
\pm 0.05 \\
\pm 0.09\end{array}$ & $\begin{array}{r}0.09 \\
0.08 \\
\pm 0.06\end{array}$ & $\begin{array}{r}-0.09 \\
\pm 0.21 \\
0.18 \\
\pm 0.07 \\
\pm 0.08\end{array}$ & $\begin{array}{r}0.18 \\
\pm 0.14 \\
-0.11 \\
-0.14 \\
\pm 0.05\end{array}$ & $\begin{array}{r}0.06 \\
\pm 0.30\end{array}$ & $\begin{array}{r} \pm 0.20 \\
0.26 \\
\pm 0.21 \\
\\
0.05\end{array}$ & $\begin{array}{l}-0.12 \\
\pm 0.07 \\
\pm 0.25 \\
\pm 0.13 \\
-0.06\end{array}$ & $\begin{array}{l}-0.05 \\
\pm 0.21\end{array}$ & $\begin{array}{r}0.13 \\
\pm 0.23 \\
-0.16 \\
\pm 0.10 \\
\pm 0.08\end{array}$ \\
\hline \multicolumn{13}{|l|}{$W(9,10,11,12)$} \\
\hline $\begin{array}{l}5 d_{z^{2}} \\
5 d_{x z} \\
5 d_{x^{2}-y^{2}} \\
5 d_{y z} \\
5 d_{x y} \\
6 s\end{array}$ & 0.08 & 0.06 & $\begin{array}{r} \pm 0.05 \\
0.14\end{array}$ & $\begin{array}{r}-0.08 \\
0.09 \\
\pm 0.07\end{array}$ & $\begin{array}{l}-0.17 \\
-0.13 \\
\pm 0.10 \\
\pm 0.11\end{array}$ & $\begin{array}{r}-0.05 \\
\pm 0.06 \\
-0.15 \\
\pm 0.25 \\
\pm 0.06\end{array}$ & $\begin{array}{l}-0.11 \\
\pm 0.05 \\
\pm 0.09 \\
\pm 0.26\end{array}$ & $\begin{array}{r}-0.09 \\
\pm 0.24 \\
0.06 \\
\pm 0.06 \\
\pm 0.08\end{array}$ & $\begin{array}{r}0.07 \\
\pm 0.05 \\
0.10\end{array}$ & $\begin{array}{r}-0.08 \\
\pm 0.18 \\
0.12\end{array}$ & $\begin{array}{l}-0.15 \\
\pm 0.19 \\
-0.20 \\
\pm 0.07\end{array}$ & $\begin{array}{l}-0.08 \\
\pm 0.11 \\
-0.11 \\
\pm 0.28 \\
\pm 0.10\end{array}$ \\
\hline
\end{tabular}


TABLE VIII. Totally symmetric molecular orbitals for the $\mathrm{W}_{9} \mathrm{H}$ surface "molecule" where hydrogen is bonded at a $5 \mathrm{CN}$ site.

\begin{tabular}{|c|c|c|c|c|c|c|}
\hline $\begin{array}{l}\text { Molecular orbital } \\
\text { energy }(e V)\end{array}$ & $\begin{array}{c}\Psi_{1}^{\prime} \\
(-13.22)\end{array}$ & $\begin{array}{c}\Psi_{2}^{\prime} \\
(-11.75)\end{array}$ & $\begin{array}{c}\Psi_{3}^{\prime} \\
(-10.42)\end{array}$ & $\begin{array}{c}\Psi_{4}^{\prime} \\
(-9.96)\end{array}$ & $\begin{array}{c}\Psi_{5}^{\prime} \\
(8.62) \\
\end{array}$ & $\begin{array}{c}\Psi_{6}^{\prime} \\
(7.36)\end{array}$ \\
\hline \multicolumn{7}{|l|}{$\mathrm{H}$} \\
\hline $1 s$ & 0.44 & 0.29 & -0.04 & -0.11 & -0.13 & 0.17 \\
\hline \multicolumn{7}{|l|}{$\mathrm{W}$} \\
\hline $\begin{array}{l}5 d_{z^{2}} \\
6 s\end{array}$ & $\begin{array}{l}0.19 \\
0.16\end{array}$ & $\begin{array}{r}0.38 \\
-0.17\end{array}$ & 0.15 & 0.10 & -0.11 & 0.54 \\
\hline \multicolumn{7}{|l|}{$\mathrm{W}(2,3,4,5)$} \\
\hline $\begin{array}{l}5 d_{z^{2}} \\
5 d_{x z}(2,5) \\
5 d_{x^{2}-y^{2}} \\
5 d_{y z}(3,4) \\
6 s\end{array}$ & $\begin{array}{l} \pm 0.08 \\
\pm 0.12\end{array}$ & $\begin{array}{l}-0.11 \\
\pm 0.15 \\
-0.11\end{array}$ & $\begin{array}{l}-0.23 \\
\pm 0.25\end{array}$ & $\begin{array}{r}0.09 \\
\pm 0.35 \\
\pm 0.35\end{array}$ & $\begin{array}{l}-0.08 \\
\pm 0.05\end{array}$ & \pm 0.16 \\
\hline \multicolumn{7}{|l|}{$\mathrm{W}(6,7,8,9)$} \\
\hline $\begin{array}{l}5 d_{z^{2}} \\
5 d_{x z} \\
5 d_{y z} \\
5 d_{x y} \\
6 s\end{array}$ & 0.06 & -0.11 & $\begin{array}{r}0.21 \\
\pm 0.10 \\
\pm 0.10\end{array}$ & -0.25 & $\begin{array}{r}0.25 \\
\pm 0.28 \\
\pm 0.28 \\
0.08\end{array}$ & $\begin{array}{l} \pm 0.12 \\
-0.05\end{array}$ \\
\hline
\end{tabular}

\section{ACKNOWLEDGMENT}

We are indebted to Professor K. Ruedenberg for helpful criticism and discussion.

${ }^{1}$ A. J. Bennett, B. McCarroll, and R. P. Messmer, Surf. Sci. 24, 191 (1971).

${ }^{2}$ J. C. Robertson and C. W. Wilmsen, J. Vac. Sci. Technol. 8, 53 (1971).

${ }^{3}$ J. C. Robertson and C. W. Wilmsen, J. Vac. Sci. Technol. 9, 901 (1972).

${ }^{4}$ D. S. M. Fassaert, H. Verbeek, and A. Van Der Avoird, Surf. Sci. 29, 501 (1972).

${ }^{5}$ A. J. Bennett, B. McCarroll, and R. P. Messmer, Phys. Rev. B 3, 1397 (1971).

${ }^{6}$ P. J. Estrup and J. Anderson, J. Chem. Phys. 45, 2254 (1966).

${ }^{7}$ P. W. Tamm and L. D. Schmidt, J. Chem. Phys. 51, 5352 (1969).

${ }^{8}$ T. E. Madey and J. T. Yates, Jr., Structure et Proprietes des Surface des Solides (Editions du Centre National de la Recherche Scientifique, Paris, 1970), No. 187, p. 155.

${ }^{9}$ K. Yonehara and L. D. Schmidt, Surf. Sci. 25, 238 (1971).

${ }^{10}$ J. T. Yates, Jr. and T. E. Madey, J. Vac. Sci. Technol. 8, 63 (1971).

${ }^{11}$ E. W. Plummer and A. E. Bell, J. Vac. Sci. Technol. 9, 583
(1972).

${ }^{12}$ R. Hoffmann, J. Chem. Phys. 39, 1397 (1963).

${ }^{13}$ G. V. Gibbs, M. M. Hamil, S. J. Lavisnathan, L. S. Bartell, and H. Yow, Am. Mineral. 57, 1578 (1972).

${ }^{14} \mathrm{M}$. Wolfsberg and C. Helmholtz, J. Chem. Phys. 20, 837 (1952).

${ }^{15}$ R. S. Mulliken, J. Chem. Phys. 23, 1833 (1955).

${ }^{16}$ H. Basch, A. Viste, and H. B. Gray, Theor. Chim. Acta 3, 458 (1965)

${ }^{17}$ F. Herman and S. Skillman, Atomic Structure Calculations (Prentice-Hall, Englewood Cliffs, NJ, 1960).

${ }^{18}$ G. Burns, J. Chem. Phys. 41, 1521 (1964).

${ }^{19}$ L. L. Lohr, Jr., and W. N. Lipscomb, Inorg. Chem. 3, 22 (1964).

${ }^{20}$ W. Lotz, J. Opt. Soc. Am. 60, 206 (1970)

${ }^{21}$ F. A. Cotton and C. B. Harris, Inorg. Chem. 6, 376 (1967).

${ }^{22}$ L. Pauling, The Nature of the Chemical Bond (Cornell U. P. Ithaca, NY, 1960), 3rd ed.

${ }^{23}$ L. F. Mattheiss, Phys. Rev. 139, A1893 (1965).

${ }^{24}$ R. L. Park, in The Structure and Chemistry of Solid Surfaces, edited by G. A. Somorjai (Wiley, New York, 1969), p. 28-1.

${ }^{25} \mathrm{~K}$. Ruedenberg and C. Edmiston, Rev. Mod. Phys. 35, 457 (1963).

${ }^{26}$ R. Gomer, R. Wortman, and R. Lundy, J. Chem. Phys. 26, 1147 (1957). 